\title{
New technology and devices for fast remote detection of alpha and beta radioactive contamination
}

\author{
Andrey Simakov, Boris Gurkovskiy, Vladimir Miroshnichenko, Evgeniy Onishchenko, Anastasiya Strutinskaya
}

\begin{abstract}
The paper summarizes the results of studies by the authors of the gas-discharge method for the fast remote registration of alpha and beta radioactive contaminants on the surfaces of a complex profile and in limited volumes of workplaces of radiation-hazardous objects. Specific features of technical solutions ensuring the detection stability of alpha and beta radiation with the help of a gas-discharge counter open to the air are considered. The design variants and main characteristics of search devices are given that allowing quickly (less than 3 seconds) detecting spot and distributed alpharadioactive contamination at distances of about $50 \mathrm{~cm}$. The design and operation modes of the portal pedestrian radiation monitor recording small alpha and beta radioactive contamination on clothes and open parts of a person's body are shown.
\end{abstract}

Keywords—alpha-radioactivity, beta-radioactivity, remote registration, ionization of air, light air ions, gas discharge method, ion track, counting mode, portal radioactive monitor

\section{Introduction}

The actuality of this work is related to the continuously growing alpha and beta radioactive contamination of the environment, caused by: a) widespread use and loss of radioactive substances in science, technology and medicine; b) radiation accidents and catastrophes (Chernobyl, Fukushima); c) decomposition and utilization of nuclear reactors; d) military actions - the use of armor-piercing shells by uranium cores, etc. A separate modern threat is radiation terrorism - the possibility of using a "dirty" bomb for mass destruction of people. A radiation hazard (toxicity) of alpha-radioactive nuclides is most pronounced when ingested in the respiratory tract and lungs. When evaluating the effective dose, the absorption coefficient for alpha particles is assumed to be 20, while for gamma quanta of all energies it is 1 . Beta-active nuclides have a markedly lower radiation toxicity, but are dangerous not only when ingested, but also with external effects on the skin, the ocular lens (radiation dermatitis, radiation cataract, etc.). The complexity of detecting alpha-radiation contamination lies in the small range of alpha particles in the air. The track of alpha particles with an energy of $10 \mathrm{MeV}$ in the air does not exceed $10 \mathrm{~cm}$, and for $2 \mathrm{MeV}$ it is not more than $2 \mathrm{~cm}$.

Andrey Simakov, National Research Nuclear University "MEPHI"(NRNU MEPHI), Moscow, Russian Federation.

Boris Gurkovskiy, NRNU MEPHI, Moscow, Russian Federation

Vladimir Miroshnichenko, , NRNU MEPHI, Moscow, Russian Federation

Evgeniy Onishchenko, NRNU MEPHI, Moscow, Russian Federation

Anastasiya Strutinskaya , NRNU MEPHI, Moscow, Russian Federation
Most advanced detectors and indicators are devices of direct action, i.e. they register the alpha particle directly on its run. These are the different types of semiconductor, gas, scintillation detectors. With the high accuracy of measurements, they can not be called remote, and they do not allow to quick investigation in large areas and objects with a complex shape. Various methods of remote registration based on secondary effects of alpha decay are being developed. For example, registration of accompanying gamma and secondary neutron radiation is widely used [1]. However, for most alpha-active nuclides, the gammaquantum yield is negligible and, for example, $0.3 \mathrm{~g}$ of $239 \mathrm{Pu}$ plutonium or $10 \mathrm{~g}$ of uranium can be registered in this way at a distance of $100 \mathrm{~cm}$. This means that "smaller" contaminants will simply not be detected, which poses a serious danger to human health. Registration for secondary neutron radiation is even more problematic for the same reason of low sensitivity.

The ionization of air by alpha and beta particles is another secondary effect widely used in radiometry. The alpha particle creates on its air track a dense aeroion cluster. Electrons create ionization distributed along the track, and their track is more than two orders of magnitude larger. The processes of the ionization-relaxation of air molecules lead to the radioluminescence, for example, it was shown in [2] that for light-emitting alpha particles, light generation is 19 \pm 3 photons per $1 \mathrm{MeV}$. Studies of specific alpha-radiation imaging devices based on radio luminescence show that, despite considerable efforts concentrated in this area, it is not yet possible to approach practical applications of such devices [3-5].

The light air ions arising during ionization of air have a sufficient long lifetime (tens of seconds), which allows them to be transported to measuring systems. This allowed the Los Alamos National Laboratory (USA) to develop a method and instruments for a remote detection of alpha radiation from the measurement of the ionization current in the air gap [6,7]. Light ions, in this case, are transported by means of an electric field, or collected on an electret electrode. The method does not allow to identifying the type of radiation and to providing a search mode operation in the field conditions.

The authors of this publication developed a practical method of gas-discharge recording, which has an order of magnitude greater sensitivity in comparison with the approaches mentioned above. In the proposed method, light ions that appear on tracks of alpha and beta particles in the air are transported by a specially formed air stream into a gas discharge chamber that is open to the air, where an avalanche discharge occurs on the high-voltage thread anode of the counting detector. The results of earlier studies and development of experimental instruments and systems based on the gas-discharge method, in particular: a) a manual search indicator "Alpha-A" serving as a "mine detector" of spot and distributed alpha - radiation contamination; 
b) pedestrian portal monitor for operative remote control of alpha and beta radioactive contamination of the surface of clothing, head and hands of people, in particular personnel of radiation hazardous objects. The results of these researchers and developments are summarized in this publication.

\section{Airionic registration based on a gas-discharge counter}

The airoion method of registration is illustrated in Fig. 1 on an example of a block diagram of an alpha-radioactivity detector block with a gas-discharge counter opened on air.

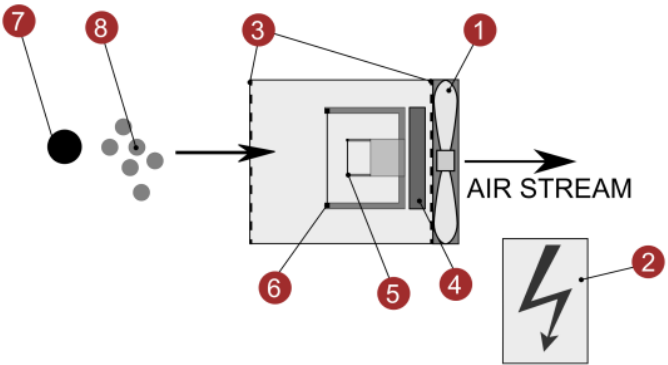

Figure 1. Aero-discharge detector unit: (1) - fan; (2) precision high-voltage source; (3) - filters; (4) - reading electronics; (5) - anode; (6) - cathode; (7) - source of alpha radiation; (8) - light air ions.

An alpha particle emitted from a radiation source (7) at an energy of $5 \mathrm{MeV}$ creates on the track about $1.6 \times 10^{5}$ pairs of carriers. These carriers basically recombine, however, some of the electrons that go beyond the critical radius $\mathrm{e}^{2} / \mathrm{kT}$ surrounding the positive ion avoid recombination and can form negative ions. They arise as a result of the attachment of an electron to a neutral air molecule or as a result of the polar dissociation of neutral molecules. The predominant electronegative impurity in the air is oxygen. The capture of an electron by oxygen proceeds at an electron energy from 2 to $4 \mathrm{eV}$. To the molecular ions formed in this way, neutral molecules of gases, which are part of the air, join under the action of polarization forces. As a result, molecular complexes (clusters) of light airoions are formed (8). The air flow generated by the fan (1) collects light air ion clusters and transfers them to a gas discharge chamber consisting of a cylindrical cathode (6) and an anode wire (5), in which a proportional gas discharge occurs. Reading electronics (4) provides the generation and calculation of output pulse signals. The operation of the detector in counting mode provides a number of advantages over the ion current measurement mode, namely: greater selectivity against the background of other types of ionization, greater sensitivity and a rapid response to the excess of the radioactive background [10]. Figure 2 shows the mechanisms of the formation of pulses in the counter from ion clusters inherent in alpha ionization and distributed beta ionization. The oscillograms of these pulses for different types of ionization are shown in Fig. 3. The differences on the oscillograms in the pulse images with different types of ionization made it possible to realize a new way of identifying radiation sources. For this purpose, based on a neural computer network, a system for automated recognition of radiation type has been proposed and practically realized. The structural scheme of the neural network is shown in Fig. 4.

An important parameter of the detector system, which determines the stable registration of radiation, is the regulated high voltage at the anode of the gas-discharge counter. It is about $3 \mathrm{kV}$ and is maintained in the proportional mode of the voltage-current characteristic (VAC) with high accuracy $(\sim 0.1 \%)$, which is provided by a precision controlled high-voltage source (2).

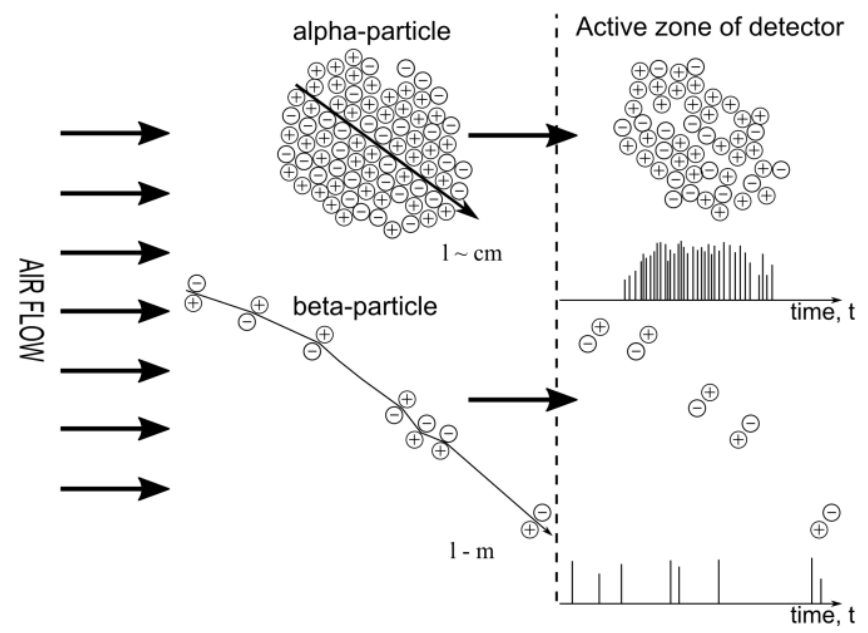

Figure 2. Mechanisms of pulses formation by alpha and beta ionization.

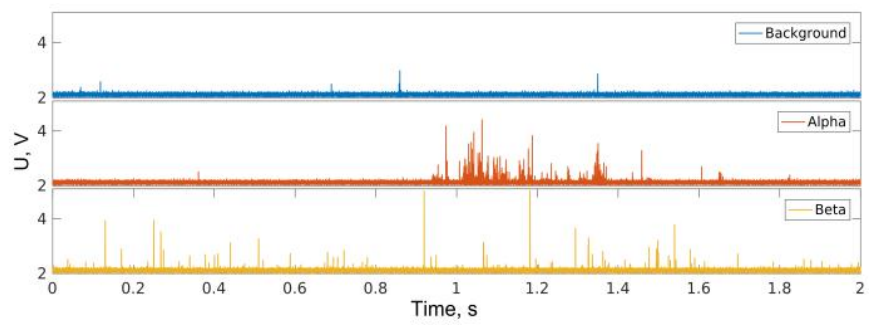

Figure 3. Oscillograms of pulses at the amplifier output for various ionization sources over a short time interval.

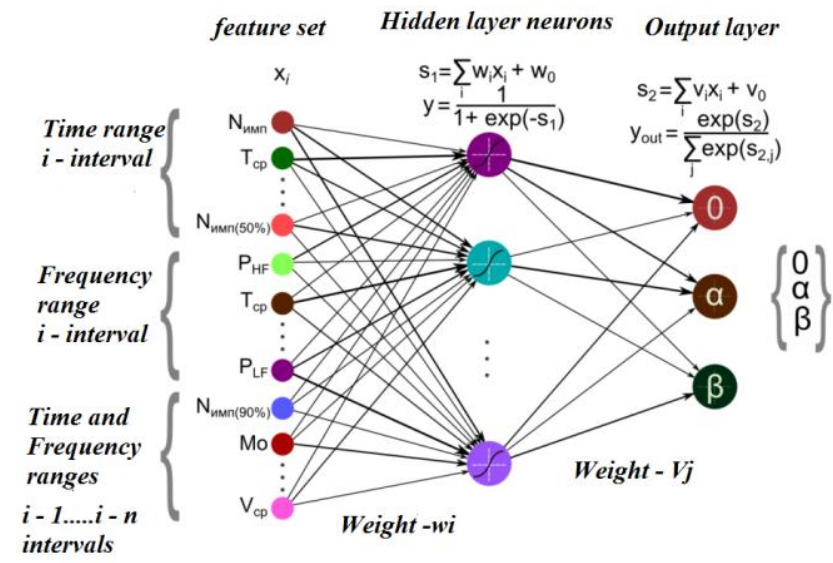

Figure 4. Structural - the scheme of the neural network for the classification of events.

The voltage-current characteristic of a gas discharge is shown in Fig. 5, where a narrow working region of the gasdischarge chamber is marked - a region of limited proportionality. In this mode, controlled gas amplification of the signal is supported on one side, on the other hand the discharge does not grow into a non-linear Geiger mode. For 
Proc. of The Eighth Intl. Conf. On Advances in Applied Science and Environmental Technology - ASET 2018 Copyright (C) Institute of Research Engineers and Doctors, USA. All rights reserved.

ISBN: 978-1-63248-155-9 doi: 10.15224/978-1-63248-155-9-15

practical implementation of the operating mode, periodic calibration of the detector unit is performed. As a result of calibration by the alpha-radioactivity standard source, a special voltage regulator sets the operating voltage on the anode of the counter and maintains it. As a special voltage regulator, a custom integrated microcircuit was used, specially developed by the authors of the article for gas detectors [11]. Its structural scheme and a photograph of topology are shown in Fig. 6 a, b.

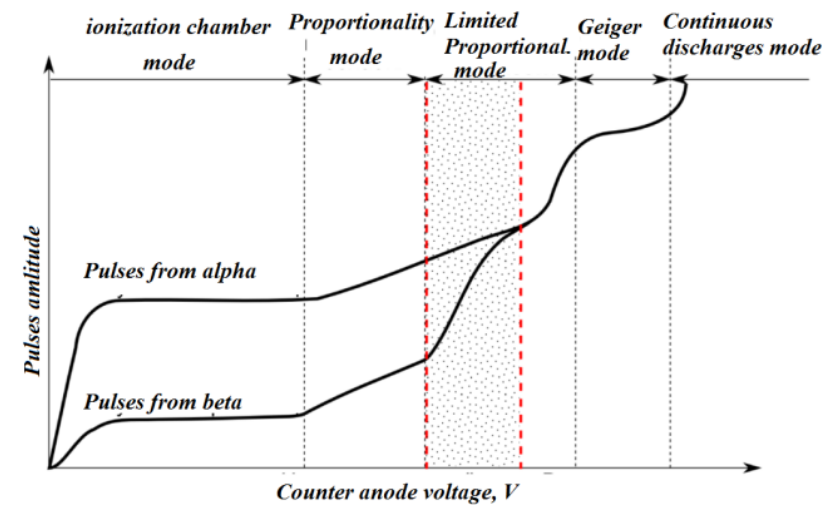

Figure. 5. Volt-ampere characteristic of the gas-discharge counter.

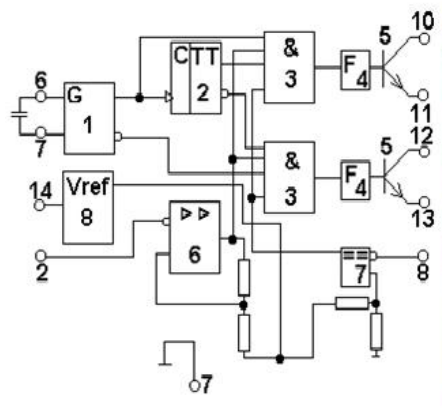

a)

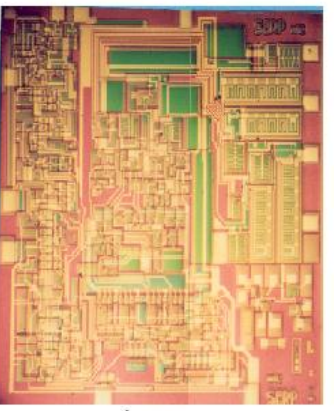

b)
Figure. 6. a) Block diagram and b) Topology of the integrated microcircuit of a controlled high-voltage supply voltage regulator for gas-discharge detectors.

The integral high-voltage stabilizer has the following characteristics:

- Primary supply voltage - + $15 \pm 3 \mathrm{~V}$;

- Output current - $5 \mathrm{~mA}$;

- Stabilized output voltage - $1.5 \ldots 4 \mathrm{kV}$

- The accuracy of the voltage stabilization (at $3 \mathrm{kV}$ ) is $0.1 \%$;

- $\quad$ Operating temperature range $-50 \ldots .+70^{\circ} \mathrm{C}$;

It should be also noted that the performance of the stabilizer is guaranteed to be preserved up to a total absorbed dose of $\gamma$ radiation of the order of $10^{6} \mathrm{rad}(\mathrm{Si})$. Therefore, the detector can operate in large radiation fields.

The detector unit is protected from dust by special replaceable fabric filters (3), especially at working in the field conditions.

\section{Designs and characteristics of specific devices}

Special designs of gas-discharge detectors have been developed for use in search instruments to detect dots or distributed sources of alpha-radioactive contamination both in the field conditions (grass, windbreak, trees, pits, etc.) and in industrial rooms (complex surfaces, slits, pipes, etc.). A block diagram and a photograph of one of such multi-tubes design are shown in Fig. 7 a, b.

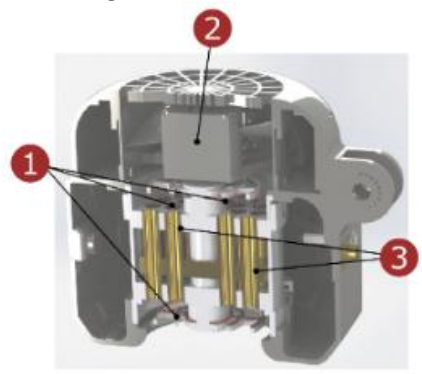

a)

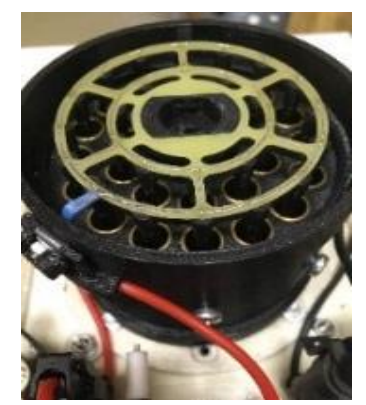

b)

Figure 7: a) Block diagram of the multi-channel detector unit (1 - gold-plated anodes, 2 - fan, 3 - gold-plated cathodes), b) picture of the block.

On the basis of this detector block several variants of search detecting devices have been developed, the photos of which are shown in Fig. 8 a, b, c. In the photographs, the devices are displayed in the hands of the operator, while working in the search mode.

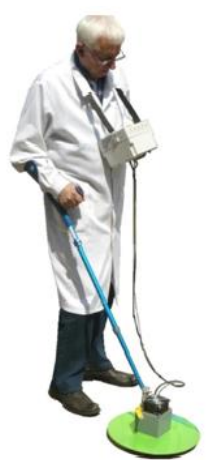

a)

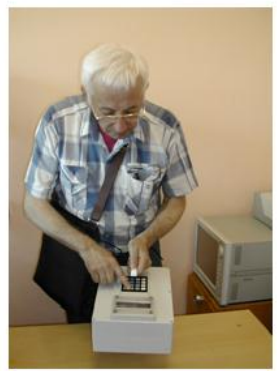

b)

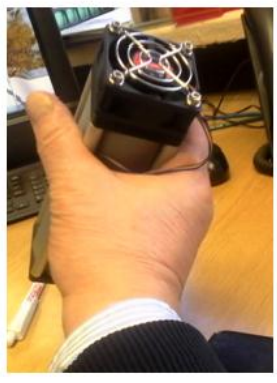

c)
Figure 8. Different designs of search mode devices in operator's hand.

More visible information about search device, which shown in Fig. 8 a, has been placed in [12].

For the examination of significant areas in the field, a detector system was developed on a mobile platform (cart). It is equipped with a powerful battery, and an additional fan, which pushes the air flow under the bottom of the cart. This makes it possible to increase the monitored area of a single measurement, and, therefore, to reduce the survey time of a particular site. A photograph of a laboratory sample of the detector system is shown in Fig. 9, and the block diagram in Fig. 10. 


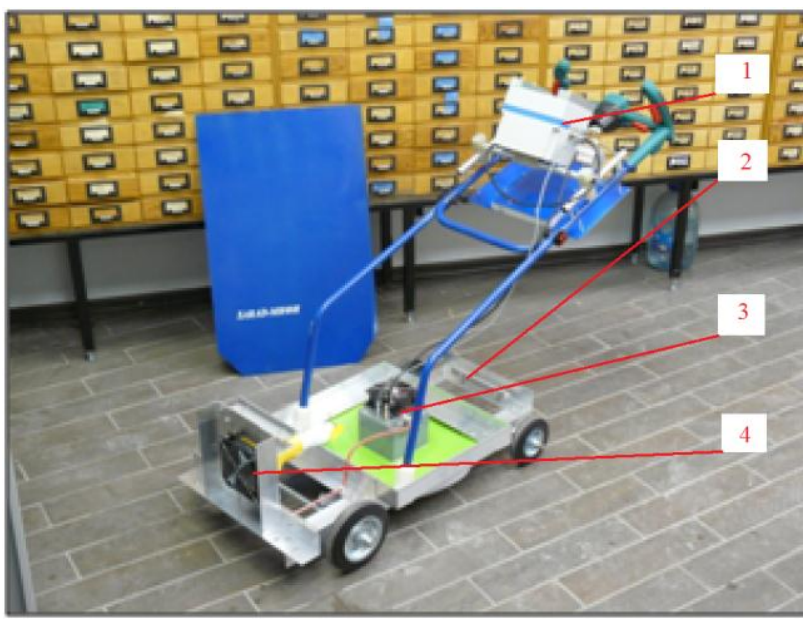

Figure 9. Picture of the detector system on the wheeled platform: 1) control unit; 2) the battery; 3) the detection unit; 4) additional fan.

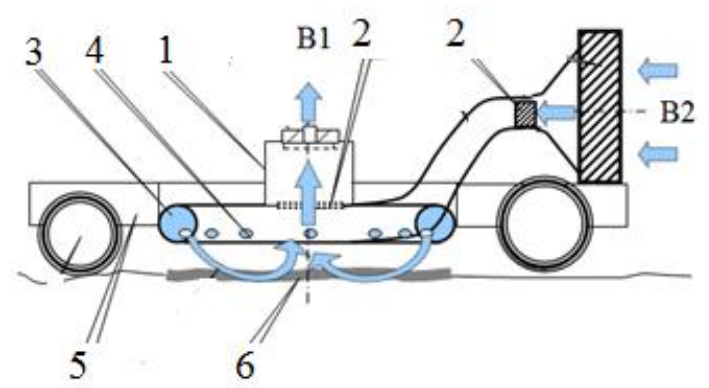

Figure 10. Block diagram of the wheeled detector system:

B1 and B2 fans, forming air flows; 1 - detector block (shown in Figure 7); 2 - air filters; 3 - annular duct; 4 - holes in the duct; 5 - wheel platform (trolley); 6 - section of the surface to be surveyed.

The search instruments shown in Figures 8 (a) and 9 have the following main characteristics:

- Detection threshold and survey area with uniform contamination: $0.01 \mathrm{~Bq} / \mathrm{cm}^{2}$ (diameter $-72 \mathrm{~cm}$;

- Detection threshold for spot contamination of the surface: $10 \mathrm{~Bq}$;

- Detection distance from the input window of the detector - 0, $5 \mathrm{~m}$;

- Selectivity of registration with associated beta and gamma radiation is $90 \%$.

- Single measurement - less than $10 \mathrm{sec}$.

The known commercial available portal monitor TwoStep $^{\mathrm{TM}}$-Gas II of the Mirion Company can detect contamination of the whole body, but it uses the technology that is based on a flow proportional counter. It features a good efficiency of the beta particles' detection, but for the registration of alpha particles, the subject needs to be close to the detector.

We developed the experimental unit of pedestrian portal monitor on basis of open-air gas-discharge counter. It can detect much smaller amounts of alpha and beta radioactive nuclides than the best of the currently used stationary radiation monitors on the basis of gamma-ray scintillation detectors. The block-diagram of this new installment is shown in Fig. 11, and picture of experimental unit demonstrated Fig. 12.

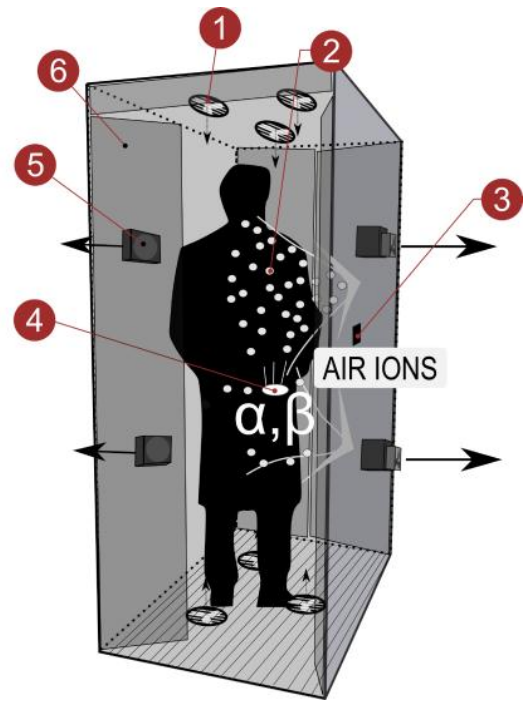

Figure 11. The block-diagram of alpha and beta portal monitor: 1 - fans; 2 - aeroions from alpha and beta particles; 3 - environmental sensors; 4 - surface sources of alpha and beta contamination; 5 -detectors; 6 -doors.

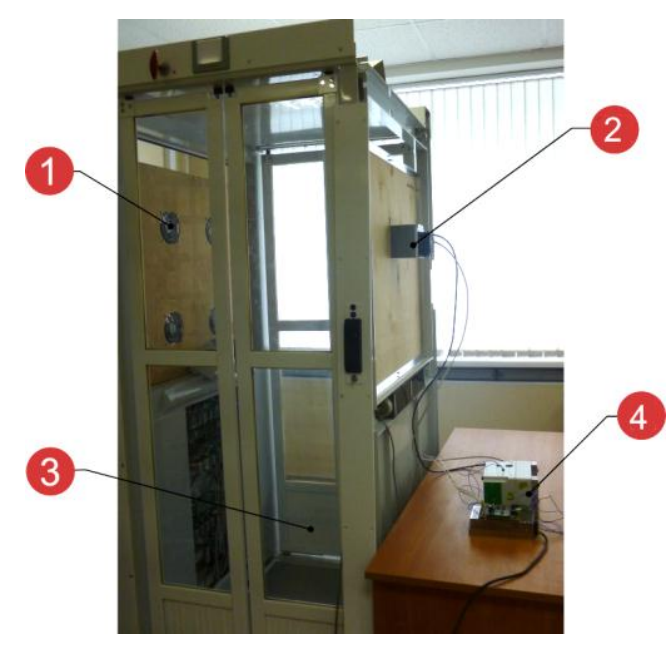

Figure 12. Picture of experimental unit of portal radiation monitor: 1 - group fans; 2 - detector; 3 -doors; 4 - control and processing block.

All mentioned above principles and instruments of gasdescharge method were used in this design. The detector system is collibrated in atomatical mode by two standard radiation source: 1 calibration - 241Am source (500 Bq); 2 calibration - $\alpha$-source $239 \mathrm{Pu}(760 \mathrm{~Bq})$. Special trained neural network was developed for radiation type identification. The same standard sources are used for neural network learning and it provides accuracy of $85-95 \%$ for identification of ionizing radiation. The experimental radiation portal allows to measure spot contamination less than $20 \mathrm{~Bq}$ on open human parts (body, hands, hair ) at 1015 seconds examination.

\section{Conclusion}

The new technology of long-distance alpha and beta radiation detection on basis of gas-discharge method allowed designing the series of new devices and systems for radiation monitoring, including environmental investigation 
Proc. of The Eighth Intl. Conf. On Advances in Applied Science and Environmental Technology - ASET 2018

Copyright (C) Institute of Research Engineers and Doctors, USA. All rights reserved.

ISBN: 978-1-63248-155-9 doi: 10.15224/978-1-63248-155-9-15

and control. The search devices has unique technical and exploitation characteristics, allowing like mine - detector in real time with high sensitivity and selectivity to detect place and to estimate alpha-radioactive contamination on complex irregular surfaces and into hard-to-reach spots.

The new technical solutions implemented in the pedestrian portal monitor, when people are examined at special checkpoints and checkpoints of civilian objects and activities such as transport, financial, customs and other administrative and public institutions, mass sports and cultural events should prevent unauthorized movement through such points -radioactive substances and reduce radiation terroristical threats.

\section{References}

[1] Richard A. Craig, Richard S. Seymour. "Detection of alpha-particle Emitting Isotopes,” USA Patent No US 7,973,287 B2 on Jul. 5, 2011.

[2] J. Sand. "Imaging of alpha emitters in a field environment," Nuclear Instruments and Methods in Physics Research Section A., 2015, vol. 782 , pp. 13-19.

[3] J. Sand, S. Ihantola. Radioluminescence yield of alpha particles in air. // New Journal of Physics, 2014, vol. 16, pp. 053022 - 053027.

[4] O.P. Ivanov, V.E. Stepanov, S.V. Smirnov, A.G. Volkovich "Development of method for detection of alpha contamination with using UV-camera "DayCor" by OFIL", 2011, IEEE Nuclear Science Symposium Conference Record, Oct. 23-29, 2011; (DOI: 10.1109/NSSMIC.2011.6154448)
[5] A.J. Crompton, K.A. Gamage. "UVC Detection as a Potential for Alpha Particle Induced Air Fluorescence Localisation". Proceedings of 15th International Conference on Environmental Science and Technology, 31августа - 2 сентября 2017 [Rhodes, Greece]); cest2017_00676.

[6] M.A.Wolf, J.L.McAtee, W.P.Unrih. "New Method Permits of Alpha Emitters at a Distance". 1990 IEEE Transactions on Nuclear Science, vol. 37, N.2, pp. 883-884.

[7] D. W. MacArthur, K. S. Allander, J. a. Bounds, M. M. Catlett, R. W. Caress, and D. a. Rutherford, "Long-range alpha detector (LRAD) technology, results, and applications," 1992, IEEE Conf. Nucl. Sci. Symp. Med. Imaging, pp. 110-112..

[8] B.V. Gurkovskiy, V.P. Miroshnichenko, E.M. Onishchenko, A.B. Simakov, T.G.Streil. "Long-distance detection of alpha-radioactivity: Method and device", Journal of Environmental Radioactivity, 2015, vol. 149, pp. 150-157. (DOI:10.1016/j.jenvrad.2015.07.019).

[9] B. Gurkovskiy, Yu Bocharov, A Simakov "Method for recognizing alpha and beta radioactive contamination in portal monitor using open-air gas-discharge counter", 2017, Proceedings of Workshop on Environmental, Energy, and Structural Monitoring Systems (EESMS). (DOI:10.1109/EESMS.2017.8052695).

[10] B. Gurkovskiy, V. Miroshnichenko, E.Onishchenko, A. Simakov, Th. Streil. "The Remote Detection of Alpha-Radioactive Nucleus Decay', Abstract of ARIS 2014, Tokyo, 1- 6 June, p. 273. [Advances in Radioactive Isotope Science].

[11] V.P. Miroshnichenko, E.M. Onishchenko, A.B. Simakov. "Monolithic precision stabilizer for gas-discharge multi-wire detector of alpha-radioactivity", Book of Abstracts of $4^{\text {th }}$ European Conference on Protection against Radon at Home and at Work, 2004, Prague, Czech Republic, June 28 - July 2, p. 59.

[12] https://www.youtube.com/watch?v=8oVQ7YJILR8; 